\title{
EL MÉTODO DE LAS CURVAS DE CALENTAMIENTO PARA LA DETERMINACIÓN DEL PODER CALÓRICO DEL BIOGÁS.
}

\section{THE METHOD OF THE HEATING CURVES FOR THE HEAT VALUE DETERMINATION OF THE BIOGAS.}

\author{
MSc. Odlanier Blanco Jorge*, PhD. Francisco García Reina**, MSc. Zulima Díaz \\ Montes* y MSc. Francisco Hernández Rodríguez** \\ * Centro de Investigaciones en Bioalimento (CIBA). Grupo de Biogás. \\ Carretera de Patria Km. 2, Mórón, Ciego de Ávila, Cuba. \\ Teléfono: 53-52806112. \\ E-mail: esp_laboratorio2@cibacav.cu \\ ** Universidad de Ciego de Ávila (UNICA), Facultad de Ciencias Técnicas, Grupo de \\ Investigación en Energía. \\ Carretera de Morón Km 9, Ciego de Ávila, Cuba. \\ Teléfono: 53-33-225702, ext. 1060. \\ E-mail: pancho30011918@gmail.com.
}

Resumen: El biogás es conocido desde el surgimiento de la humanidad, pero no ha sido hasta ahora, impulsado por las necesidades económicas y el cambio climático, que ha despertado gran interés su uso con fines energéticos, hoy los conocimientos de esta tecnología se amplían y son los países en vías de desarrollo los más interesados. Por esta causa se ha empleado en el Centro de Investigaciones de Bioalimentos un digestor anaerobio de cúpula fija de primera generación a escala de laboratorio para controlar los parámetros de funcionamiento $(\mathrm{pH}$, temperatura, frecuencia y volumen de alimentación, presión) y así aplicar el método empírico de las curvas de calentamiento para determinar el poder calórico relativo del biogás, obtenido a partir de diferentes sustratos, demostrando que el biogás posee una concentración de metano superior al 50 $\%$ y que el residual del cerdo Criollo Cubano, en comparación con cerdos descendiente de raza $\mathrm{CC} 21$, mejora las cualidades energéticas del biogás aumentando en un $9 \%$ el poder calórico relativo $(4496.13 \mathrm{~kJ} / \mathrm{kg}$ de biogás) y la velocidad media de calentamiento $(0.009 \mathrm{~K} / \mathrm{s})$. Las mediciones realizadas poseen un alto grado de confiabilidad con un coeficiente de determinación R2 $=0.99$ y los resultados poseen un error no mayor del $1 \%$.

Palabras clave: digestión anaerobia, biogás, poder calórico.

Abstract: Biogas is known since the emergence of humanity, but has not been so far,
driven by economic needs and climate change, which has aroused great interest its use for
energy purposes, today the knowledge of this technology is expanded and are the
developing countries most interested. For this reason, an anaerobic digester of fixed dome
of first generation at laboratory scale has been used in the Bioaliments Research Center to
control the operating parameters (pH, temperature, frequency and volume of feed,
pressure) and thus apply the method Empirical of the heating curves to determine the
relative caloric power of the biogas, obtained from different substrates, showing that the
biogas has a methane concentration higher than 50\% and that the residual of the Cuban 
Criollo pig, in comparison with pigs descendant of race $\mathrm{CC} 21$, improves the energy qualities of biogas by increasing the relative caloric power by $9 \%(4496.13 \mathrm{~kJ} / \mathrm{kg}$ of biogas) and the average heating rate $(0.009 \mathrm{~K} / \mathrm{s})$. The measurements made have a high degree of reliability with a coefficient of determination $\mathrm{R} 2=0.99$ and the results have an error no greater than $1 \%$.

Keywords: anaerobic digestion, biogas, heat.

\section{Introducción:}

Los combustibles fósiles son fuentes no renovables de energía y su recuperación tarda largos períodos de años. Su uso constituye un reto, fundamentalmente para los países subdesarrollados, pues se encuentran concentrados en determinadas regiones del planeta y su precio es cada vez más elevado debido a su escasez y al mercado especulativo relacionado con este recurso (Ortiz, Wainshtok \& Hernández, 2016).

La sobreexplotación de esta energía fósil, unida a su condición de ser un recurso limitado, está cuestionando cada día más el que pueda seguir siendo, por sí sola, la fuente para la producción de la energía del futuro, teniendo en cuenta también las condicionantes de tipo económico y técnico de la explotación de los nuevos yacimientos. Si a este factor se suma que las energías de origen fósil son las principales responsables de las emisiones de gases de efecto invernadero y por tanto del calentamiento global, se comprende el hecho de que desde hace algún tiempo, a nivel mundial, se hayan vuelto las miradas hacia los programas de desarrollo de fuentes de energías renovables, que permiten cubrir parte de la demanda futura en condiciones económicas viables (Miembros de la mesa del Biogás, 2010).

El Centro de Investigaciones de Bioalimentos (CIBA), ubicado en el municipio de Morón, desarrolla, dentro de sus líneas de trabajo, el empleo de los digestores, no solo en la remoción de los residuales, sino también, en el uso de la energía como valor agregado de esta tecnología. La entidad cuenta con 2 prototipos a escala convencional, uno tubular de polietileno y otro de cúpula fija construido de concreto con capacidades para procesar un volumen de materia orgánica de 3.7 $\mathrm{m}^{3} /$ día y $54 \mathrm{~m}^{3} /$ día respectivamente, constituyendo un centro de referencia dentro de la provincia en el aprovechamiento de las fuentes renovables de energía (FRE).

Según lo referido por Huerga (2013), en estas tecnologías se presentan dificultades de origen físico o constructivo, biológico o de manejo y otros. Estos inconvenientes influyen de una u otra manera en el rendimiento energético del biogás siendo los de origen biológicos los más importantes debido a que, dicho rendimiento, depende en gran medida de la calidad del sustrato incorporado y al buen equilibrio biológico dentro del digestor, determinando la concentración de metano, responsable de las propiedades energéticas del biogás. Debido a la inestabilidad en algunos parámetros de manejo (frecuencia y volumen de alimentación) y la carencia de una tecnología apropiada (Cromatógrafo de gases o Calorímetro de JUNKERS), se desconoce la eficiencia energética del biogás obtenido, lo que limita el aprovechamiento de este combustible en todo su potencial.

\section{Materiales y métodos:}

\section{- Método de Curvas de Calentamiento.}

La base teórica del método de las curvas de calentamiento se encuentra en la Ley de Enfriamiento de Newton descrita por Holman (1999), la cual plantea que el gradiente de temperatura con respecto al tiempo, cuando la presión, la temperatura y el volumen permanecen constantes, establece una correlación lineal con el calor trasmitido, o lo que es lo mismo, con el poder calórico de la sustancia combustionada.

Por consiguiente, el método consiste en quemar, en idénticas condiciones de presión y temperatura, un volumen de biogás (cuyo poder calórico se quiere determinar) y el mismo volumen de un gas cuyo poder calórico se conoce. Ambos volúmenes se queman calentando un volumen de agua por vez, después de obtenidos los datos de temperatura $(T)$ en grados Celsius y tiempo ( $t$ ) en segundo se procede, mediante el programa estadístico Mathcad versión 15.0, a la construcción de la curva de calentamiento $\mathrm{T}\left({ }^{\circ} \mathrm{C}\right) \mathrm{v} / \mathrm{s} \mathrm{t}(\mathrm{s})$, posteriormente se determinan, para cada relación de datos, los valores de incremento de la temperatura $(\Delta \mathrm{T})$ y se correlacionan con los diferentes tiempos (ti).

Cada curva realizada, según el número de repeticiones, permite obtener igual número de 
pendientes, calculando el promedio de dichas pendientes se obtiene la velocidad media de calentamiento $(\mathrm{K} / \mathrm{s})$, con el poder calórico del gas conocido se puede calcular el poder calórico del gas incógnita (Martina, García, Corace, Aeberhard \& Valentín, 2006).

\section{- Descripción del ensayo.}

Consiste en combustionar a presión constante de $108 \mathrm{~mm}$ en la columna de agua, varios gases combustibles, obtenidos en un digestor anaerobio de cúpula fija a escala de laboratorio y almacenado en un gasómetro de baja presión sin flotante, ambos, desarrollados como medio experimental por Blanco (2018) (ver figura 1), y situar a calentar a una altura de $0.011 \mathrm{~m}$ del borde superior del mechero Bunsen un Erlenmeyer de área total igual a $0.024 \mathrm{~m}^{2}$ con $0.1 * 10^{-3} \mathrm{~m}^{3}$ de agua destilada (patrón) a la cual se le medirá $\mathrm{pH}$ y temperatura inicial, permitiendo medir el aumento de temperatura del agua destilada con respecto al tiempo y definir la curva de calentamiento, conociendo la pendiente de dicha curva y utilizando métodos matemáticos se determina la velocidad media de calentamiento o el poder calórico relativo del gas combustible empleado.

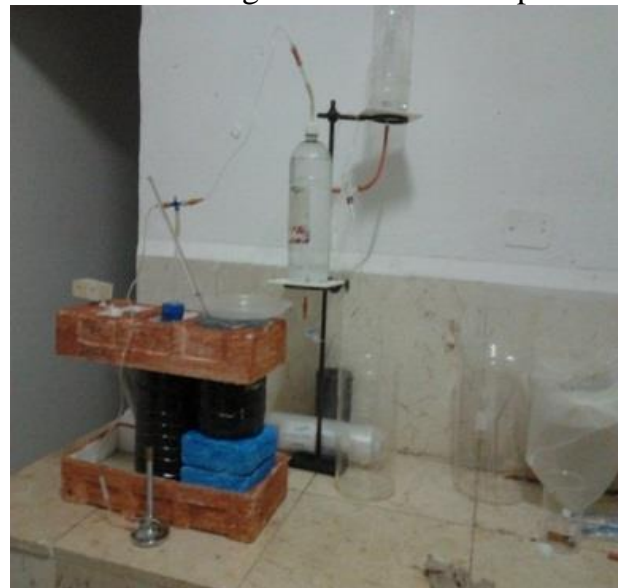

Fig. 1- Digestor anaerobio con gasómetro empleado en las mediciones.

Después de establecidas las condiciones iniciales, el ensayo será desarrollado en varias etapas:

1ra etapa: será analizado el gas natural presurizado obteniendo un grupo de mediciones, que servirán de patrón y serán comparadas con las obtenidas en los ensayos con biogás.

El ensayo con el biogás será desarrollado en diferentes momentos, propiciando analizar el biogás obtenido a partir de diferentes condiciones del sustrato y su origen. 2da etapa: Se empleará el biogás producido por el digestor convencional, el sustrato empleado es generado por cerdos comerciales (Sus scropha domésticus) descendientes de raza $\mathrm{CC} 21$, de diferentes categorías y diluido con agua común o de consumo del CIBA. Esto permitirá evaluar las características energéticas del biogás que se recibe en la cocina del centro.

3ra etapa: Se intentará reproducir las condiciones anteriores pero a escala de laboratorio para evaluar las diferencias entre los resultados anteriores y los obtenidos en estas condiciones. Por tanto el biogás analizado será obtenido en el digestor a escala de laboratorio a partir del residual generado por cerdos comerciales (Sus scropha domésticus) descendientes de raza $\mathrm{CC} 21$, con categoría de ceba sometidos a una dieta específica la cual consiste en un volumen de $3 \mathrm{~kg} /$ día/animal de alimento (15\% soya, $50 \%$ maíz, $35 \%$ miel C). Este sustrato será diluido para alimentar el biodigestor, en una proporción excreta/agua (1:3) según lo descrito en el epígrafe 2.1. El agua empleada en la dilución del sustrato será agua común o de consumo procedente de la fuente de abasto del CIBA, la cual será sometida a un grupo de análisis organolépticos y físico-químicos, los resultados obtenidos serán comparados con los valores permisibles establecidos por la N/C 827:2017 Agua PotableRequisitos Sanitarios, evitando la incorporación en exceso de sustancias inhibidoras o perturbadoras de las rutas metabólicas en la digestión anaerobia.

4ta etapa: Después de comparados los resultados anteriores se procederá a probar otras alternativas de sustrato.

Por tanto el biogás analizado será obtenido a partir del residual generado por cerdos (Sus scropha domésticus) descendientes de razas de cerdo Criollo Cubano con categoría de ceba, sometidos a una dieta específica dividida en dos partes, la primera consiste en un volumen de $3 \mathrm{~kg} /$ día/animal de alimento (15\% soya, $50 \%$ maíz, $35 \%$ miel C) y la otra parte consiste en un volumen de $0.30 \mathrm{~kg}$ de CT_115 (Pennisetun purpureum cv. CT_115), Girasolillo (Tithonia diversifolia (Hemsl.) A Gray) y residuos de comida del comedor obrero, además por las características de crianza, su dieta esta enriquecida por caracoles de la familia Planorbidae y otras especies como insectos, larvas y lombrices, según lo descrito por Rodríguez, Pérez \& Castro (2014). Este sustrato será diluido para alimentar el biodigestor, en una proporción excreta/agua (1:3) según lo descrito en el epígrafe 2.1. El agua empleada posee el mismo origen de la etapa 
anterior por lo que será sometido al mismo tratamiento.

\section{-Principales equipos de medición.}

Los principales equipos utilizados fueron (por orden), el analizador de gases (Multitec 540. SEWERIN), la balanza analítica, el termómetro de mercurio y un cronómetro digital (Android).
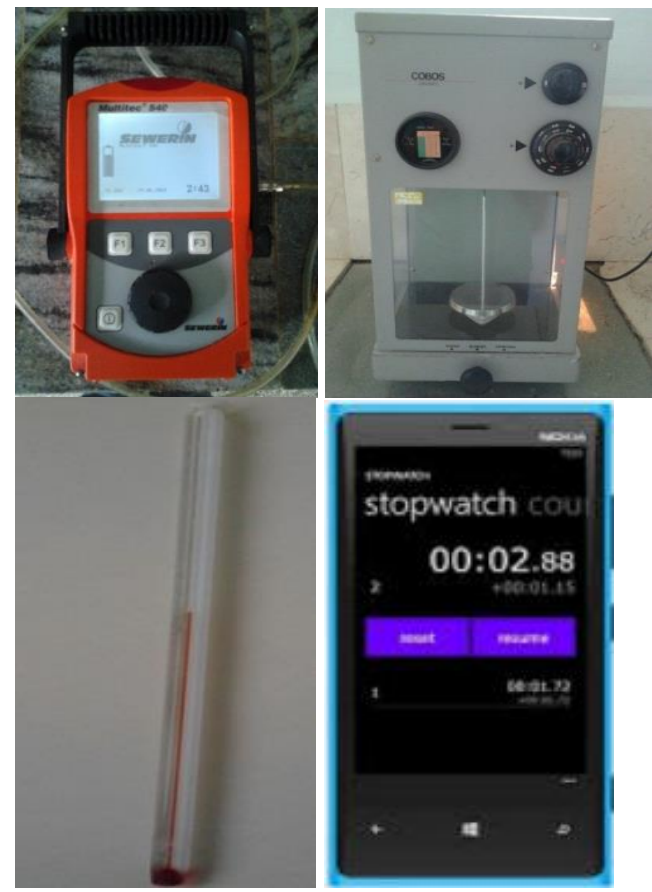

Fig. 2- Equipos de medición utilizados.

\section{Resultados y su discusión:}

\section{-Determinación de la Velocidad media de calentamiento.}

\section{Gas natural presurizado.}

El porciento de metano en el gas natural presurizado es de un $100 \%$ aproximadamente, lo que será importante pues el resultado obtenido será empleado como punto de comparación entre dicho gas y el biogás, el pH del agua destilada es el mismo y la temperatura ambiente se encuentra entre $24{ }^{\circ} \mathrm{C}$ y $25^{\circ} \mathrm{C}$.

Mediciones realizadas:

Inmediatamente de establecidas las condiciones iniciales del ensayo se sucedieron las 4 mediciones de la variación de temperatura $(\mathrm{T})$ con respecto al tiempo (t), a presión y volumen constante.

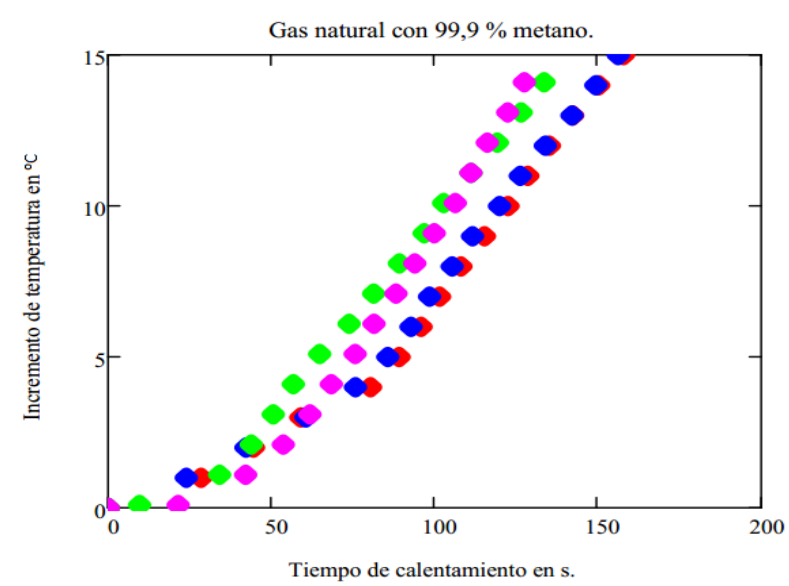

Fig. 3 Curvas de calentamiento del gas natural presurizado.

Posterior a la construcción de las curvas de calentamiento (figura 3) se tomaron los valores de las mediciones y se calculó el incremento de la temperatura con respecto al tiempo, posibilitando la construcción de una gráfica de incremento (figura 8-b), de la cual se obtendrá la velocidad de calentamiento.

De la media de las pendientes se obtiene la velocidad de calentamiento.

Velocidad media de calentamiento $0.110 \mathrm{~K} / \mathrm{s} \pm 1 \%$

\section{Digestor convencional con sustrato de cerdo CC21.}

El porciento de metano en el biogás, obtenido a partir de sustrato del cerdo comercial, en el digestor convencional es de un $55 \%$, los resultados derivados de las mediciones posibilitarán evaluar las propiedades energéticas del biogás, el pH del agua destilada es el mismo y la temperatura ambiente se encuentra entre $24^{\circ} \mathrm{C}$ y $25^{\circ} \mathrm{C}$.

Mediciones realizadas:

Inmediatamente de establecidas las condiciones iniciales del ensayo se sucedieron las 4 mediciones de la variación de temperatura $(\mathrm{T})$ con respecto al tiempo (t) a presión y volumen constante.

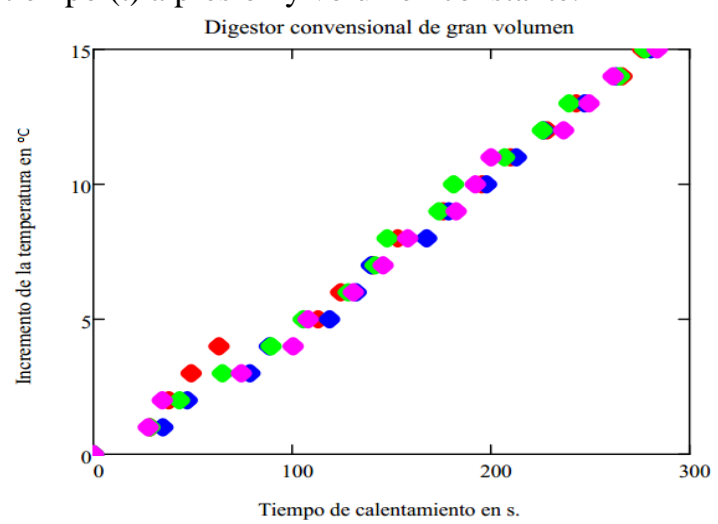

Fig. 4 Curvas de calentamiento del biogás analizado. 
Posterior a la construcción de las curvas de calentamiento (figura 4) se tomaron los valores de las mediciones y se calculó el incremento de la temperatura con respecto al tiempo, posibilitando la construcción de una gráfica de incremento (figura 9-b), de la cual se obtendrá la velocidad de calentamiento.

De la media de las pendientes se obtiene la velocidad de calentamiento.

Velocidad media de calentamiento $0.055 \mathrm{~K} / \mathrm{s} \pm 1 \%$

\section{Digestor de laboratorio con sustrato de cerdo CC21.}

El porciento de metano en el biogás, obtenido a partir de sustrato del cerdo comercial, del digestor a escala es de un $51 \%$ aproximadamente, los resultados derivados de las mediciones posibilitarán evaluar las propiedades energéticas del biogás en cuestión, el pH del agua destilada se mantiene en un rango de 6 y la temperatura ambiente se encuentra entre $24^{\circ} \mathrm{C}$ y $25^{\circ} \mathrm{C}$.

Mediciones realizadas:

Inmediatamente de establecidas las condiciones iniciales del ensayo se sucedieron las 4 mediciones de la variación de temperatura $(\mathrm{T})$ con respecto al tiempo (t) a presión y volumen constante, empleando los instrumentos y materiales de laboratorio mencionados en el epígrafe 2.4 y 2.5 respectivamente.

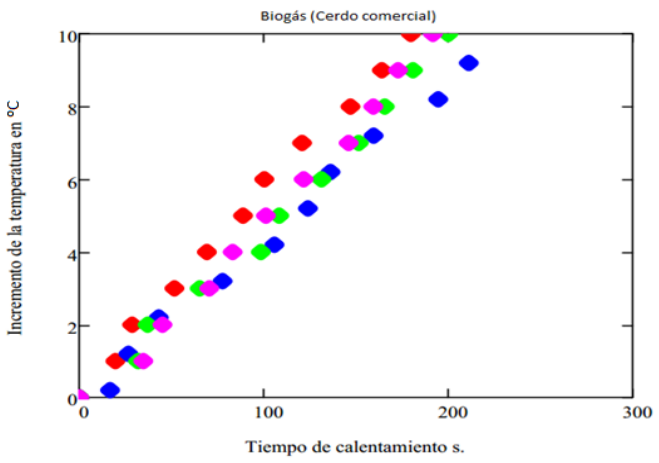

Fig. 5 Curvas de calentamiento del biogás analizado.

Posterior a la construcción de las curvas de calentamiento (figura 5) se tomaron los valores de las mediciones y se calculó el incremento de la temperatura con respecto al tiempo, posibilitando la construcción de una gráfica de incremento (figura 6), de la cual se obtendrá la velocidad de calentamiento.

De la media de las pendientes se obtiene la velocidad de calentamiento.

Velocidad media de calentamiento $0.051 \mathrm{~K} / \mathrm{s} \pm 1 \%$

\section{Digestor de laboratorio con sustrato de cerdo Criollo Cubano.}

El porciento de metano en el biogás, obtenido a partir de sustrato del cerdo comercial, del digestor a escala es de un $59 \%$ aproximadamente, los resultados derivados de las mediciones posibilitarán evaluar las propiedades energéticas del biogás en cuestión, el pH del agua destilada se mantiene en un rango de 6 y la temperatura ambiente se encuentra entre $21^{\circ} \mathrm{C}$ y $25^{\circ} \mathrm{C}$.

Mediciones realizadas:

Después de establecidas las condiciones iniciales del ensayo se realizaron las 4 mediciones de la variación de temperatura $(\mathrm{T})$ con respecto al tiempo (t) a presión y volumen constante, empleando los instrumentos y materiales de laboratorio mencionados en el epígrafe 2.4 y 2.5 respectivamente.

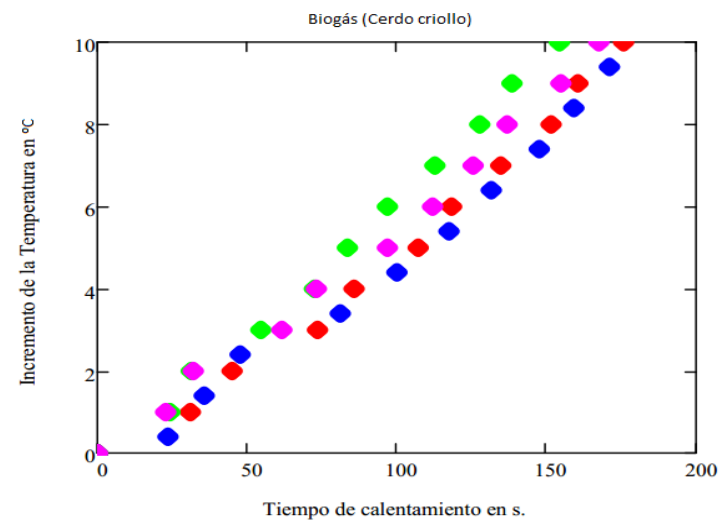

Fig. 6 Curvas de calentamiento del biogás analizado.

Posterior a la construcción de las curvas de calentamiento (figura 6) se tomaron los valores de las mediciones y se calculó el incremento de la temperatura con respecto al tiempo, posibilitando la construcción de una gráfica de incremento, de la cual se obtendrá la velocidad de calentamiento.

De la media de las pendientes se obtiene la velocidad de calentamiento.

Velocidad media de calentamiento $0.059 \mathrm{~K} / \mathrm{s} \pm 1 \%$

\section{Discusión de los resultados.}

El autor propone el método empírico de las curvas de calentamiento (Temperatura $\mathrm{v} / \mathrm{s}$ tiempo) implementado en el desarrollo de la investigación, el mismo resulta sencillo, dada las condiciones económicas y la carencia tecnológica, y posibilita la determinación de las propiedades energéticas (velocidad media de calentamiento y poder calórico) de los gases combustibles empleados.

El análisis estadístico evidencia la confiabilidad de las mediciones realizadas, las cuales presentan una alta linealidad entre el tiempo y la temperatura de calentamiento con un coeficiente de determinación 
de $\mathrm{R}^{2}=0.99$ y un error del $1 \%$. Por otro lado la determinación de la desviación estándar muestra la poca dispersión de los datos al mostrar valores medios de Stdev=0.030.

\section{Poder calórico relativo del biogás.}

Empleando el poder calórico del gas natural presurizado para un $100 \%$ de $\mathrm{CH}_{4}$ y las fracciones de las concentraciones de $\mathrm{CH}_{4}$ en los diferentes biogás analizados se puede determinar el poder calórico relativo de cada biogás según se muestra en la tabla 5.

Tabla 1- Poder calórico relativo de los gases analizados.

\begin{tabular}{|c|c|c|}
\hline Sustancia & $\begin{array}{c}\text { Composición } \\
\text { media } \mathrm{CH}_{4}(\%)\end{array}$ & $\begin{array}{c}\text { Poder calórico } \\
\text { relativo }(\mathrm{kJ} / \mathrm{kg})\end{array}$ \\
\hline Gas Natural presurizado & 100 & 49957 \\
\hline $\begin{array}{c}\text { Digestor convencional } \\
\text { (cerdo comercial) }\end{array}$ & 55 & 27476.35 \\
\hline $\begin{array}{c}\text { Digestor laboratorio } \\
\text { (cerdo comercial) }\end{array}$ & 50 & 24978.5 \\
\hline $\begin{array}{c}\text { Digestor laboratorio } \\
\text { (œrdo criollo) }\end{array}$ & 59 & 29474.63 \\
\hline
\end{tabular}

\section{Discusión de resultados.}

De acuerdo con los resultados expuestos se puede decir que los biogás estudiados pueden ser empleados como fuente de energía en varias actividades (cocción de alimentos, alumbrado doméstico y conservación de granos), a criterio del autor, el poder calórico aún es insuficiente para algunas actividades como el accionamiento de motores de la industria automotriz y la generación de electricidad, por lo que se recomienda emplear tecnologías de purificación, posibilitando eficiencia en el proceso de combustión del biogás.

Los resultados obtenidos demuestran que los residuos generados por el cerdo Criollo Cubano aumentan el poder calórico relativo y la velocidad media de calentamiento del biogás. Por lo que resulta una ventaja para nuestro país motivar a los productores a la crianza de estos animales.

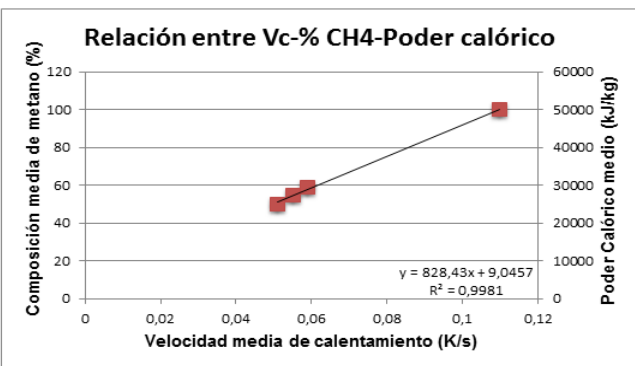

Fig. 7- Correlación de los diferentes biogás.

Discusión de los resultados.
La representación gráfica de los resultados obtenidos (figura 7), demostró la interrelación que existe entre el porciento de metano y el poder calórico relativo de un gas determinado, con su velocidad media de calentamiento obtenida a partir de las pendientes del incremento de la temperatura con respecto al tiempo. Además, permite determinar el porcentaje de metano y el poder calórico relativo de cualquier otro biogás, siempre que sean igualadas las condiciones experimentales, sin llegar a ser importante el sustrato empleado para su producción.

\section{Conclusiones}

1- La implementación del método de las curvas de calentamiento permitió determinar el poder calórico relativo de los biogás estudiados, con valores de $27476.35 \mathrm{~kJ} / \mathrm{kg}$ (digestor convencional con sustrato de cerdo CC21), $24978.5 \mathrm{~kJ} / \mathrm{kg}$ (digestor laboratorio con sustrato de cerdo CC21) y $29474.63 \mathrm{~kJ} / \mathrm{kg}$ (digestor laboratorio con sustrato de cerdo Criollo Cubano) y la interrelación que existe entre el porciento de metano y el poder calórico relativo, con la velocidad media de calentamiento.

2- Las características de alimentación del cerdo Criollo Cubano posibilita que su residual mejore las cualidades energéticas del biogás, aumentando en un $9 \%$ el poder calórico relativo $(4496.13 \mathrm{~kJ} / \mathrm{kg}$ de biogás) y en $0.009 \mathrm{~K} / \mathrm{s}$ la velocidad media de calentamiento, en comparación con los cerdos CC21.

3- La mediciones de la temperatura con respecto al tiempo, presentan un coeficiente de determinación medio de $\mathrm{R}^{2}=0.99$ y los resultados un error no mayor del $1 \%$.

\section{Bibliografía.}

1- Blanco, O. (2018). Diseño y construcción de un digestor anaerobio a escala de laboratorio para el estudio del biogás con fines energéticos. Eco Solar 61 de la Sociedad Cubana para la Promoción de las Fuentes Renovables de Energía y el Respeto Ambiental, Cubasolar. (ISSN: 1028-6004)

2- Holman, J. P. (1999). Transferencia de calor. Octava edición (primera en español). España: Concepción Fernández Madrid (ISBN 007844785-2).

2- Huerga, I. (2013). Biodigestor para el tratamiento de los efluentes del sector porcino. Ricardone, Santa Fe: Escuela Agrotécnica $\mathrm{N}^{\circ}$ 383 - Julio Luis Maiztegui.

3- Martina, P., García, E., Corace, J., Aeberhard, M. \& Valentín, A. (2006). Biogás e Isobutano: análisis comparativo de una propiedad 
termodinámica. Argentina: Grupo de Investigación en Energías Renovables (GIDER) - Departamento de Termodinámica Facultad de Ingeniería - UNNE.

4- Miembros de la mesa de biogás (2010). El sector del biogás agroindustrial en España. Madrid, España: Dirección General de Recursos Agrícolas y Ganaderos y Ministerio de Medio Ambiente y Medio Rural y Marino.

5- Ortiz, M., Wainshtok, H., \& Hernández, H. (2016). Diseño de un biodigestor de ferrocemento semisoterrado de cúpula fija. Cuba: Revista Cubana de Ingeniería. Vol. VII, No. 1, pp. 5 - 11, (ISSN 2223 -1781).

6- Rodríguez, D., Pérez, N., Castro, M. (2014). Flora y fauna endémica como alternativa para la alimentación del Cerdo Criollo Cubano, caso de estudio CIBA, Ciego de Ávila. Revista Producción Porcina Vol. 21, No 4. 